\title{
208 Fusion of MR-derived anatomical and late enhancement image data with ablation mapping for verification of lesion delivery for cardiac radio-frequency ablation
} Benjamin R Knowles ${ }^{* 1}$, Matthew Ginks ${ }^{1}$, Dennis Caulfield ${ }^{2}$,
Michael Cooklin ${ }^{2}$, Reza Razavi ${ }^{1}$, Nicholas Gall ${ }^{2}$, Tobias Schaeffter ${ }^{1}$ and Kawal Rhode ${ }^{1}$

Address: ${ }^{1}$ King's College London, London, UK and ${ }^{2}$ Guy's and St Thomas' NHS Foundation Trust, London, UK

* Corresponding author

from I I th Annual SCMR Scientific Sessions

Los Angeles, CA, USA. I-3 February 2008

Published: 22 October 2008

Journal of Cardiovascular Magnetic Resonance 2008, I0(Suppl I):A69 doi:10.1 I86/1532-429X-10-SI-A69

This abstract is available from: http://jcmr-online.com/content/I0/SI/A69

(C) 2008 Knowles et al; licensee BioMed Central Ltd.

\section{Introduction}

Cardiac arrhythmias, such as atrial fibrillation and atrial flutter, are now increasingly treated using minimally invasive catheter ablation techniques as part of electrophysiology studies (EPS). The ablation sites can be mapped using electro anatomical mapping systems (EAMS), but there is no gold standard for ablation mapping. The success rate is sometimes poor and often patients must undergo repeat ablations. To date, MRI has shown sufficient sensitivity to detect ablation lesions $[1,2]$, however MRI data has not been compared to the ablation lesions as mapped using an EAMS. In this abstract, we present the first findings for comparing MRI and EAMS ablation mapping.

\section{Methods}

We investigated a patient with atrial flutter using our hybrid x-ray and MR (XMR) imaging system that consists of a 1.5 T Philips Achieva MR system and a Philips BV Pulsera $\mathrm{x}$-ray system. Using our XMR image fusion technology we are able to fuse MR and x-ray image data [3]. Prior to EPS, the patient underwent MR anatomy imaging consisting of a whole heart 3D SSFP and a gadoliniumenhanced right-heart MRA scan. During the EPS, the ablation locations were determined from two oblique $\mathrm{x}$-ray projections and were mapped to the MRI-derived cardiac anatomy using our XMR EAMS. Post-procedure, the patient underwent MR imaging consisting of an ECG trig- gered, respiratory navigated, 3D IR-TFE late enhancement scan for the detection of the ablation lesions (image resolution $=1.3 \times 1.3 \times 2.5 \mathrm{~mm}^{3}, \mathrm{TR} / \mathrm{TE}=4.3 / 2.1 \mathrm{~ms}$, flip angle $=30^{\circ}$, inversion time set to null myocardium, 130 $\mathrm{ms}$ acquisition window). The trigger delay time was so that movement within the atria was minimised.

Offline, the late enhancement image data were thresholded, such that only the most enhancing regions were visible, and registered to the whole heart SSFP images. This allowed the enhancing regions to be visualised in the context of anatomical data. The ablation locations as determined from the XMR EAMS (figure 1d) were then superimposed using our XMR fusion technique so that the location of these could be compared to the enhancing regions (figure $1 \mathrm{a}-\mathrm{c}$ ).

\section{Results}

We noted several findings from the combined anatomical, enhancement, and XMR EAMS ablation location image data: (1) High signal intensity was observed from the late enhancement images extending from the IVC to the right atrial isthmus and then into the right ventricle - this was as expected from regions targeted by the cardiologist; (2) enhancing regions compared well with the sites of the ablations mapped with the XMR EAMS but not all ablations mapped with the XMR EAMS had a corresponding 


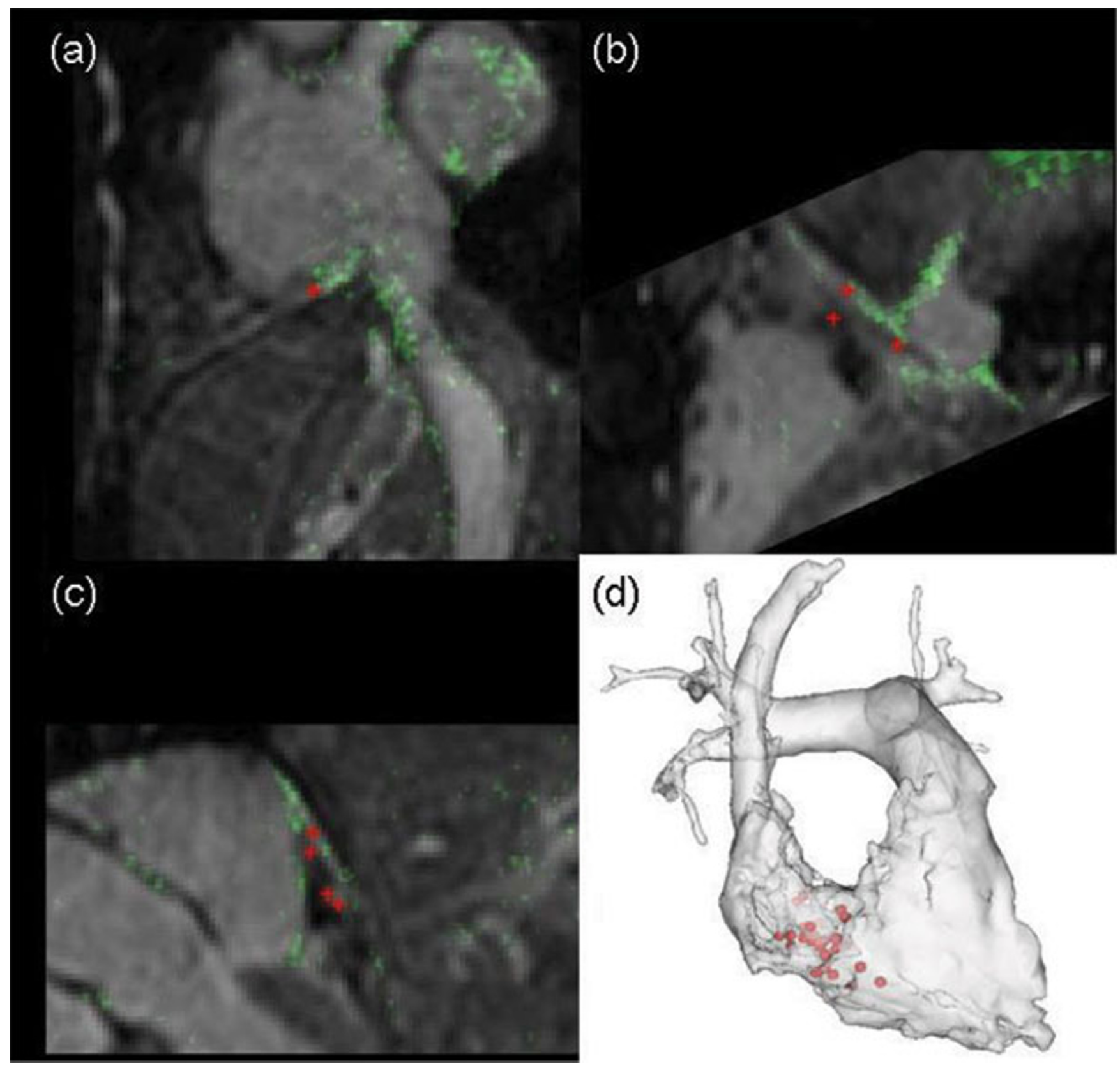

Figure I

We have developed a technique to fuse ablation lesions and cardiac anatomy from MRI with ablation maps from an electro-anatomical mapping system. We present the fused data results for a patient undergoing treatment for atrial flutter in the XMR setting. (a-c) Enhancing areas (green) seen in context of anatomical images (greyscale). Overlaid are the ablation points (red) from the XMR EAMS. (d) Ablation points (red) mapped onto the right-heart surface model derived from the gadolinium-enhanced MRA images.

region of enhancement - it may be that not all lesions were successful, i.e. not enough energy was deposited at the ablation site; and (3) enhancement was also seen in many of the great vessel walls.

\section{Discussion}

We have presented a method for fusing MRI-derived anatomical and late enhancement data with ablation location data from an EAMS. The initial patient data showed a good correlation between MRI-derived enhancement and EAMS-derived ablation location. This strategy will allow 
for validation of EAMS and the possibility for using MRI to establish complete lines of conduction block after an EPS. In the XMR setting it could be possible to use the enhancement imaging to localise and treat areas of incomplete ablation and thereby increase the success rate for cardiac ablation procedures.

\section{References}

I. Dickfeld, et al:: J Am Coll Cardiol 47(2):370-8. 2006 Jan 17

2. Peters, et al.: Radiology 2007, 243(3):690-5.

3. Rhode, et al.: IEEE Trans Med Imaging 2005, 24(I I): 1428-40.

Publish with Biomed Central and every scientist can read your work free of charge

"BioMed Central will be the most significant development for disseminating the results of biomedical research in our lifetime. " Sir Paul Nurse, Cancer Research UK

Your research papers will be:

- available free of charge to the entire biomedical community

- peer reviewed and published immediately upon acceptance

- cited in PubMed and archived on PubMed Central

- yours - you keep the copyright

Submit your manuscript here:

http://www.biomedcentral.com/info/publishing_adv.asp 